predicted by a $\mathrm{kNN}$ model that included age and CCI (figure 1).

Conclusion The k-NN algorithm is a versatile and promising tool for R0 resection in HGSOC patients, which outperforms logistic regression. The model, which is very much reflective of 'previous clinical experience' can be directly available to clinicians and is expected to improve accuracy with data expansion.

Disclosures No disclosures.

\section{SURVIVAL IMPLICATION OF PRE-TREATMENT IMAGING TUMOR DISSEMINATION PATTERN IN PATIENTS SURGICALLY TREATED FOR ADVANCED HIGH GRADE SEROUS OVARIAN CANCER}

Alexandros Laios, Yong Tan, Angelika Kaufmann, Mohamed Otify, Richard Hutson, Amudha Thangavelu, George Theophilou, David Nugent, Diederick Dejong. St James's University Hospital; Leeds Teaching Hospitals; Gynaecologic Oncology

\subsection{6/ijgc-2020-ESGO.107}

Introduction/Background Clarity and precision about the anatomical extent of disease in cancer is essential for prognostication, research, and cancer-control activities. To select effective therapeutic approaches for advanced high-grade serous ovarian cancer (HGSOC), yet the most prevalent and lethal form, it is important to identify stratification factors that could accurately predict prognosis before initial intervention. We hypothesized that women with different tumor dissemination patterns at pre-treatment imaging would have different prognosis.

Methodology This was a retrospective analysis of 209 FIGO stage III-IV HGSOC women, who were scheduled for cytoreductive surgery in SJUH Leeds between Jan 2015 to Dec 2018 with curative or life-prolonging intent. CT scans were reported by an MDT radiologist. Three pre-treatment imaging dissemination patterns were identified and verified by final histology. A Cox proportional hazard analysis was used to test the effect of imaging dissemination patterns, age, performance status (PS), timing of surgery (upfront vs delayed cytoreduction), surgical complexity score (SCS), residual disease (RD), disease score, and type of chemotherapy on survival. KaplanMeier survival curves were produced using SPSS ${ }^{\circledR} 26$.

Results There were no statistical differences in the cytoreduction rates amongst the three groups (figure 1). The mean progression free survival (PFS) for patients grouped as intraperitoneal $(n=137)$, intraperitoneal and lymphatic $(n=56)$, and intraperitoneal and haematogenous $(\mathrm{n}=16)$ was 26.5 (95\% CI 23.4-29.6), 21.3 (95\% CI 18.3-24.4) and 19.1 months (95\% CI 15.1-22.9), respectively. The mean overall survival (OS) was 45.8 (95\% CI 41.5-50.2), 34.8 (95\% CI
29.2-40.3) and 30.7 months (95\% CI 24.5-36.9), respectively $(p=0.05)$ (figure 2). The mean PFS and OS for the entire cohort was 25 months (95\% CI 22.6-27.3) and 41.8 (95\% CI 38.3-45.2), respectively. For PFS, Cox regression analysis identified PS (HR 1.23, 95\% CI 1.1-1.5, p=0.04), RD (HR $0.69,95 \%$ CI $0.46-0.98, \mathrm{p}=0.05)$ as statistically significant. For OS, Cox regression analysis identified PS (HR 1.47, 95\% CI 1.14-1.89, $\mathrm{p}=0.03$ ), dissemination pattern (HR 1.36, 95\% CI $1.02-1.86, p=0.05$ ) as statistically significant.

Conclusion For HGSOC prognosis, one should consider not only the patient's disease burden but also their overall medical status and ability to undergo extensive surgery. Prolonged survival rates were found predominantly in those patients with intraperitoneal only pre-treatment imaging dissemination pattern. Baseline tumor dissemination pattern can be a prognostic factor for overall survival. Classification of such patterns can help counsel patients initially on their prognosis and identify those who might benefit from intraperitoneal chemotherapy.

Disclosures No disclosures.

\section{CONTRASTING CLINICAL CHARACTERISTICS AND TREATMENT PATTERNS IN WOMEN WITH NEWLY DIAGNOSED ADVANCED-STAGE OVARIAN CANCER IN AUSTRALIA, SOUTH KOREA AND TAIWAN}

${ }^{1}$ Hung-Hsueh Chou, ${ }^{2}$ Anna Defazio, ${ }^{3}$ Byoung-Gie Kim, ${ }^{4}$ Chih-Long Chang, ${ }^{5}$ David Bowtell, ${ }^{6}$ Heng-Cheng Hsu, ${ }^{5}$ Nadia Traficante, ${ }^{3}$ Soo Young Jeong, ${ }^{7}$ Wen-Fang Cheng, ${ }^{5}$ Sian Fereday. ${ }^{1}$ Cgmh-Lk Chang Gung Medical Foundation, Linkou Branch; ${ }^{2}$ Centre for Cancer Research, The Westmead Institute for Medical Research; The University of Sydney; Department of Gynaecological Oncology, Westmead Hospital; ${ }^{3}$ Samsung Medical Center, Sungkyunkwan University School of Medicine; ${ }^{4}$ Mackay Memorial Hospital; ${ }^{5}$ Peter Maccallum Cancer Centre; Sir Peter Maccallum Cancer Centre Department of Oncology, The University of Melbourne; ${ }^{6}$ National Taiwan University Hospital, Hsin-Chu Branch; ${ }^{7}$ National Taiwan University Hospital

\subsection{6/ijgc-2020-ESG0.108}

Introduction/Background Epithelial ovarian cancer (EOC) is often associated with diagnosis at an advanced stage, poor prognosis and high mortality. Limited data exist on the clinical management of ovarian cancer (OC) patients in the AsiaPacific region. We evaluated secondary databases from Australia, South Korea and Taiwan to review the current standard of care in a real world setting prior to the introduction of poly-(adenosine diphosphate-ribose) polymerase inhibitor (PARPi) maintenance after first-line chemotherapy.

Methodology Data from medical records of nearly 1,000 women diagnosed with advanced-stage EOC in a 5 year period, between January 2014 and December 2018 were obtained from clinic- (Taiwan, South Korea) and cohort-based
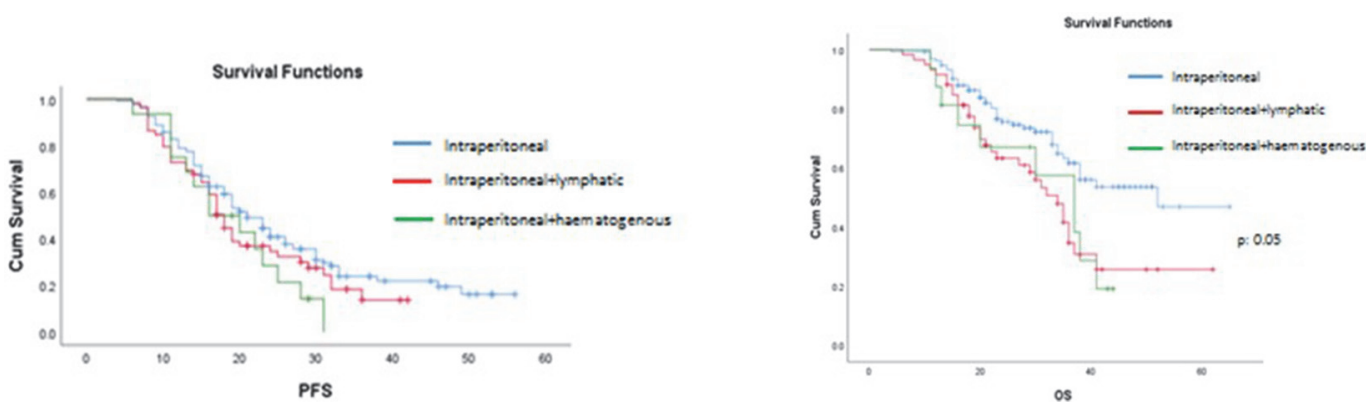

Abstract 250 Figure 2 
(Australia) OC registries. Patients on first-line maintenance with PARPis were excluded. Treatment characteristics including the use of neoadjuvant therapy, outcomes of cytoreductive surgery and the use of bevacizumab as maintenance were summarised with descriptive statistics. Duration of treatment, time to real world progression and any other time-to-event outcomes (e.g. overall survival, platinum based chemotherapy-free interval and time to first subsequent treatment and surgery) will be analysed using Kaplan Meier methodology.

Results Table 1 describes the demographic characteristics and treatment patterns of the study population. Overall, patients received a median of 6 cycles of primary platinum chemotherapy; complete or partial remission was the most common outcome. For patients with data on progression, the median time to first progression in months (two sided 95\% confidence interval) was 16.2 (14.6-18.1), $17.9(16.2-19.3)$ and 17.2 (14.7 19.7) in Australian, South Korean and Taiwanese patients, respectively. From the currently available dataset of patients with high grade serous type investigated for germline BRCA1/2 mutations: 30/169, 17.8\% (Australia), 80/354, $22.6 \%$ (South Korea) and 7/21, 33.3\% (Taiwan) of patients tested positive for germline BRCA1/2 mutations.

Abstract 257 Table 1 Demographic characteristics and treatment patterns in epithelial ovarian cancer

\begin{tabular}{lccc}
\hline Parameters & $\begin{array}{c}\text { Australia } \\
(\mathbf{N}=\mathbf{2 2 3})\end{array}$ & $\begin{array}{c}\text { South Korea } \\
(\mathbf{N}=\mathbf{5 1 3})\end{array}$ & $\begin{array}{c}\text { Taiwan } \\
(\mathbf{N = 1 2 0})\end{array}$ \\
\hline Median age, years & 65.2 & 56.0 & 59.5 \\
$\begin{array}{l}\text { Median weight, kgs } \\
\text { High-grade serous tumour, \% }\end{array}$ & 70.0 & 55.6 & 56.0 \\
$\begin{array}{l}\text { Interval debulking } \\
\text { surgery/neo-adjuvant chemotherapy, } \\
\text { \% }\end{array}$ & 82.5 & 84.6 & $100^{*}$ \\
$\begin{array}{l}\text { Platinum chemotherapy, \% } \\
\text { Bevacizumab+chemotherapy, } \mathrm{n}(\%)\end{array}$ & 56.5 & 24.0 & 9.1 \\
$\begin{array}{l}\text { Maintenance-bevacizumab, } \mathrm{n}(\%) \\
\text { Maintenance-others, } \mathrm{n}(\%)\end{array}$ & $59(29.6)$ & $84(16.4)$ & $23(19.2)$ \\
\hline *Taiwan only recruited women with high-grade serous ovarian cancer & $12(10.0)$ \\
\end{tabular}

Conclusion Although there were differences in demographics and treatment patterns, advanced-stage EOC had poor prognosis and relatively short progression-free intervals across AsiaPacific countries with well developed healthcare systems, highlighting the need to develop novel approaches to improve patient outcomes. Variation in the germline BRCA1/2 mutation rates across three datasets is probably due to differences in the composition of the contributing registries (clinic or cohort-based).

Disclosures Anna DeFazio has received a research grant from AstraZeneca.

David Bowtell has received research grants from AstraZeneca, BeiGene and Genentech Roche. David Bowtell is also a consultant for Exo Therapeutics.

Sian Fereday has received a research grant from AstraZeneca.

Nadia Traficante has received a research grant from AstraZeneca

Byoung-Gie Kim has received a research grant from AstraZeneca, Cellid and Eutilex.

Soo Young Jeong, Hung-Hsueh Chou, Chih-Long Chang, Heng-Chen Hsu and Wen-Fang Cheng have no conflict of interests to be declared

\section{SERUM MARKERS AND CYTOKINES IN PATIENTS WITH OVARIAN CANCER, ENDOMETRIOSIS OR OTHER BENIGN OVARIAN TUMOURS}

${ }^{1}$ Marek Nowak, ${ }^{2}$ Marcin Misiek, ${ }^{1}$ Łukasz Janas, ${ }^{1}$ Iwona Piwowarczyk, ${ }^{1}$ Malwina Soja, ${ }^{1}$ Martyna Masternak, ${ }^{3}$ Ewa Głowacka, ${ }^{4}$ Michał Kiełbik, ${ }^{4}$ Izabela Szulc-Kiełbik, ${ }^{4}$ Magdalena Klink. 'Department of Operative Gynecology and Gynecologic Oncology, Polish Mother's Memorial Hospital - Research Institute; ${ }^{2}$ Department of Gynaecology, Holy Cross Cancer Centre; '3aboratory Diagnostics Centre, Polish Mother's Memorial Hospital Research Institute; ${ }^{4}$ Institute of Medical Biology, Polish Academy of Sciences

\subsection{6/ijgc-2020-ESGO.109}

Introduction/Background The gold standard of serum tumour markers to detect ovarian cancer is cancer antigen 125 (CA125), human epididimis protein-4 (HE-4) and risk of ovarian malignancy algorithm (ROMA). However there is still need to improve its accuracy. Cytokines play a crucial role in tumour growth and progression according to proangiogenic and immunosuppressive acting. The aim of this study was to investigate the potential use of serum levels of selected cytokines in preoperative diagnosing of adnexal mass.

Methodology The study group consisted of 120 patients: 35 with epithelial ovarian cancer (EOC) and 85 with benign ovarian tumours (24 teratomas, 27 endometriotic and 34 other epithelial). We measured in sera obtained preoperatively the level of CA125, HE-4 and the panel of 6 cytokines: interleukin (IL) $1 \beta, 6,8,10,12$, tumour necrosis factor (TNF) using cytometric bead array (CBA) and one chemokine CXCL1/ GRO- $\alpha$ by ELISA method.

Results Serum levels of IL-6, IL-8, IL-10 and CXCL1/GRO- $\alpha$ were significantly higher in patients with ovarian cancer (2045 $\mathrm{pg} / \mathrm{ml} ; 208 ; 32 ; 356 \mathrm{pg} / \mathrm{ml}$, respectively) than in women with benign ovarian tumours $(17 \mathrm{pg} / \mathrm{ml} ; 29 ; 16 ; 127 \mathrm{pg} / \mathrm{ml}$, respectively). The similar pattern was present with standard ovarian cancer markers - CA125 (959 vs $43 \mathrm{U} / \mathrm{ml}$ ) and HE-4 (534 vs $51 \mathrm{pmol} / \mathrm{l})$. Other investigated cytokines had similar levels in all groups of patients. Analyzing the differences in the subgroups of women with benign ovarian tumours we didn't observe any significant except CA125 and IL-8; they were slightly elevated in cases of endometriotic ovarian cysts.

Conclusion Proinflammatory cytokines (IL-6, IL-8), immunosuppressive (IL-10) and CXCL1/GRO- $\alpha$ were elevated in sera of EOC patients what points on their role in cancer development. Moreover, they might be useful in preoperative differential diagnosis of ovarian tumors, as a supplemental markers especially as they were not elevated in cases of endometriosis. Disclosures All authors report no conflict of interest.

\section{REAL-WORLD-DATA ON PLATINUM OUTCOMES AFTER PARP INHIBITORS PROGRESSION IN HIGH GRADE SEROUS OVARIAN CANCER PATIENTS}

${ }^{1}$ Andrea Plaja Salarich, ${ }^{1}$ ris Teruel García, ${ }^{2}$ Beatriz Pardo Burdalo, ${ }^{2}$ Marta Gil-Martin, ${ }^{2} J o s e p$ Maria Piulats Rodriguez, ${ }^{3}$ Claudia Fina Planas, ${ }^{3}$ María Pilar Barretina Ginesta, ${ }^{4}$ Andrea Gonzalez Valencia, ${ }^{4}$ Anna Esteve Gomez, ${ }^{1}$ Margarita Romeo Marin. ${ }^{1}$ Catalan Institute of Oncology (Ico)-Badalona, Germans Trias I Pujol University Hospital (Hugtip); BadalonaApplied Research Group in Oncology (B-Argo); Medical Oncology Department; ${ }^{2}$ Catalan Institute of Oncology (Ico)-Idibell, L'hospitalet; Medical Oncology Department; ${ }^{3}$ Catalan Institute of Oncology (Ico)-Girona, Girona Biomedical Research Institute (Idibgi); Medical Oncology Department; ${ }^{4}$ Catalan Institute of Oncology (Ico)-Badalona, Germans Trias I Pujol University Hospital (Hugtip); Badalona-Applied Research Group in Oncology (B-Argo); Statistics Department

10.1136/ijgc-2020-ESGO.110 